\title{
A simple approach towards the sign problem using path optimisation
}

\author{
Francis Bursa $^{a}$ and Michael Kroyter ${ }^{b}$ \\ ${ }^{a}$ School of Physics and Astronomy, University of Glasgow, \\ Kelvin Building, University Avenue, Glasgow, G12 8QQ, United Kingdom \\ ${ }^{b}$ Department of Sciences, Holon Institute of Technology (HIT), \\ 52 Golomb St., Holon 5810201, Israel \\ E-mail: francis@semichrome.net, michaelkro@hit.ac.il
}

ABSTRACT: We suggest an approach for simulating theories with a sign problem that relies on optimisation of complex integration contours that are not restricted to lie along Lefschetz thimbles. To that end we consider the toy model of a one-dimensional Bose gas with chemical potential. We identify the main contribution to the sign problem in this case as coming from a nearest neighbour interaction and approximately cancel it by an explicit deformation of the integration contour. We extend the obtained expressions to more general ones, depending on a small set of parameters. We find the optimal values of these parameters on a small lattice and study their range of validity. We also identify precursors for the onset of the sign problem. A fast method of evaluating the Jacobian related to the contour deformation is proposed and its numerical stability is examined. For a particular choice of lattice parameters, we find that our approach increases the lattice size at which the sign problem becomes serious from $L \approx 32$ to $L \approx 700$. The efficient evaluation of the Jacobian $(O(L)$ for a sweep) results in running times that are of the order of a few minutes on a standard laptop.

KEYWORDS: Lattice field theory simulation

ARXIV EPRINT: 1805.04941 


\section{Contents}

1 Introduction 1

$\begin{array}{lll}2 & \text { Simple integration contours } & 7\end{array}$

$\begin{array}{lll}3 & \text { A general ansatz for the integration contour } & 10\end{array}$

4 The Jacobian 11

5 Results $\quad \mathbf{1 5}$

$\begin{array}{lll}5.1 & \text { Fitting the parameters } & 16\end{array}$

$\begin{array}{ll}\text { 5.2 The observables and the mean phase factor for the various contours } & 17\end{array}$

$\begin{array}{lll}5.3 & \text { Resolving the numerical instability } & 21\end{array}$

$\begin{array}{lll}6 & \text { Discussion } & 23\end{array}$

\section{Introduction}

The sign problem poses a challenge for a lattice simulation of many physical theories, ranging from widely-studied systems such as QCD and other theories with a finite chemical potential $[1,2]$, through the simulation [3] of PT-symmetric theories $[4,5]$, to the simulations [6] of more exotic systems such as string field theory [7]. It also appears in the simulations of various condensed matter theories as well as in other systems. In general, one faces a challenge whenever $e^{-S}$ is not a positive quantity. In such a case $e^{-S}$ cannot be identified as a (non-normalized) probability density and the Metropolis algorithm [8] cannot be applied without a modification. A simple modification is reweighting. However, for fast oscillations of the phase of the action the computational cost of evaluation using reweighting grows exponentially with the lattice volume. The sign problem is this exponential behaviour.

Several methods have been introduced in order to circumvent the sign problem: one could attempt to expand the problematic part of the integrand in a Taylor expansion such that all terms in the expansion involve only integrals with a constant phase [9]. While this method works for some systems, it introduces an error in the estimate of observables on top of the standard statistical error. Also, one could not expect it to be useful for large values of the parameters used in the expansion, e.g. the chemical potential. Another approach is to analytically continue the problematic parameters to values that lead to no sign problem and then continue back [10,11]. While this method could also be used in some cases, numerical analytical continuation can be quite challenging and the approach suffers from problems similar to those of the Taylor expansion approach. A third approach is the complex Langevin method [12]. Here, stochastic dynamics is used for the calculation 
of observables and there is no reference to $e^{-S}$ as a probability density. Hence, the sign problem is avoided. Nonetheless, this method also has its limitations [13-15].

An important ingredient of the complex Langevin method is the complexification of the degrees of freedom. Complexification can be used in other ways as well: one could attempt to avoid the phase oscillation causing the sign problem by using (the multi-dimensional form of) Cauchy's integral theorem for deforming the original integration contour to one without (or at least with less) phase oscillations. When using Cauchy's theorem, one must take care not to pass any singularities of the integrand and to deform the asymptotic integration range only when it vanishes fast enough, e.g. using Jordan's lemma. For most physical theories the analytical continuation of the action is regular. Hence, the first issue is of no concern for these theories. The asymptotic behaviour of the integrand, on the other hand, can become singular for specific contours. For potentials that behave asymptotically as $V \simeq \phi^{n}$ with $N$ variables ( $N$ equals the product of field components by the number of lattice points) there are generically $(n-1)^{N}$ different homology classes of integration contours $[16,17]$. One should therefore be careful not to deform the contour away from the original homology class.

A possible prescription for the contour deformation is to use Lefschetz thimbles [17], which also give the steepest descent of the (real part of the) measure. The implementation of Lefschetz thimbles in lattice simulations was introduced in [18]. Despite the success of this approach, it is also not without faults:

1. While it is known that Lefschetz thimbles are manifolds, explicit expressions defining these manifolds are absent. This leads to complicated and expensive algorithms for verifying that the integration contour does not leave the thimble.

2. While in some cases only a single thimble contribution is relevant in the continuum limit [18], in other cases one needs many thimbles. Since the mean phase factor, i.e. $\left\langle e^{i \operatorname{Im}(S)}\right\rangle_{P Q}$, the mean value of the phase in the phase quenched ensemble, can differ among different thimbles, this could lead to reemergence of the sign problem as a "global sign problem", especially in light of the fact that the number of homology classes (the number of independent thimbles) goes to infinity in the continuum limit.

3. Lefschetz thimbles are constructed in a way that keeps the imaginary part of the classical action constant. However, when working with thimbles the integration measure changes. This leads to the "residual sign problem".

4. For any given lattice there is a different set of thimbles. One should therefore identify the thimbles as well as their contribution to the desired cohomology class independently for every lattice size, lattice spacing, mass, etc. This becomes more and more complicated as the lattice size is increased.

The above issues can be summarized by noting that Lefschetz thimbles improve, but do not completely resolve the sign problem, while reducing the running cost from exponential to $O\left(V^{4}\right)$. In some cases the computational cost of a thimble simulation can be further decreased [19]. Nonetheless, it is still advisable to look for improvements and alternatives 
to the Lefschetz thimble method. Indeed, generalisations of the thimble method have been proposed, in which the above mentioned issues are addressed by choosing a single integration contour as an approximation to the sum of all the relevant thimbles [20-22].

In this work, we propose a method for a different deformation of the integration contour. We do not attempt to approximate the thimbles. Thimbles are defined by a gradient flow, which defines a real $N$-dimensional submanifold of the complex $N$-dimensional space. On the other hand, the requirement of a vanishing imaginary part puts only one real constraint and hence its solution is a $(2 N-1)$-dimensional real manifold. We intend to choose an $N$-dimensional subspace of this manifold, or an approximation to such a subspace, in order to obtain a proper integration cycle. This might appear to be too arbitrary as compared to a cycle defined by a gradient flow. However, there is much arbitrariness also in the definition of a gradient flow: in order to define the gradient operator, a metric should be introduced in the complex $N$-dimensional space. It is possible to choose this metric to be the flat metric, but this choice is not canonical [17]. The only relevant attribute of this metric is its compatibility with the complex structure, which guarantees that the imaginary part is constant along the flow, i.e. the metric must be Kähler [17]. While not all the manifolds with constant imaginary part and appropriate boundary conditions can be obtained by varying the metric (also, some metric variations lead to a reparametrisation rather than a change of the obtained manifold), some can be obtained this way. This illustrates that even "the thimble" is not a uniquely defined object. Moreover, an integration cycle which is not the thimble can be chosen to be a single contour that already takes into account the change in the integration measure. Hence, with a proper choice of integration contour, both the residual sign problem and the global sign problem could be avoided.

For illustrating our method we consider the specific case of a Bose gas at a finite chemical potential. This model is often used for the purpose of examining new methods for dealing with the sign problem, e.g. [18, 23]. On the lattice (in lattice units) the action of the model is,

$$
\begin{aligned}
S= & \sum_{k}\left(\left(d+\frac{m^{2}}{2}\right)\left(u_{k}^{2}+v_{k}^{2}\right)+\lambda \frac{\left(u_{k}^{2}+v_{k}^{2}\right)^{2}}{4}\right. \\
& \left.-\sum_{\nu=1}^{d-1}\left(u_{k} u_{k+\hat{\nu}}+v_{k} v_{k+\hat{\nu}}\right)-\cosh (\mu)\left(u_{k} u_{k+\hat{0}}+v_{k} v_{k+\hat{0}}\right)+i \sinh (\mu)\left(u_{k} v_{k+\hat{0}}-v_{k} u_{k+\hat{0}}\right)\right) .
\end{aligned}
$$

Here, $u_{k}, v_{k}$ are respectively the real and imaginary parts of a single complex field,

$$
\Phi_{k}=u_{k}+i v_{k}
$$

the index $k$ runs over all lattice sites and $d$ is the dimensionality of space-time. While the signature is Euclidean, the "time" direction is special, since the interactions related to it differ from those of the other coordinates due to the presence of the chemical potential $\mu$. Moreover, the terms related to the chemical potential are the ones responsible for the imaginary part of the action. The action enjoys a U(1) symmetry, even for a non-zero chemical potential. 
Rescaling the fields by a factor of $\sqrt{\frac{2 d+m^{2}}{\lambda}}$ the action becomes,

$$
\begin{aligned}
S= & \frac{1}{\lambda \alpha^{2}} \sum_{k}\left(\frac{u_{k}^{2}+v_{k}^{2}}{2}+\frac{\left(u_{k}^{2}+v_{k}^{2}\right)^{2}}{4}\right. \\
& \left.-\alpha\left(\sum_{\nu=1}^{d-1}\left(u_{k} u_{k+\hat{\nu}}+v_{k} v_{k+\hat{\nu}}\right)+\cosh (\mu)\left(u_{k} u_{k+\hat{0}}+v_{k} v_{k+\hat{0}}\right)+i \sinh (\mu)\left(v_{k} u_{k+\hat{0}}-u_{k} v_{k+\hat{0}}\right)\right)\right) .
\end{aligned}
$$

where we defined

$$
\alpha \equiv \frac{1}{2 d+m^{2}}
$$

For fixed $d$, this constant ranges from $\alpha=0$, which corresponds to the infinite mass limit to $\alpha=\frac{1}{2 d}$, which corresponds to the zero mass limit. For small values of the chemical potential the vacuum of the theory is in an unbroken phase of the $\mathrm{U}(1)$ symmetry, while a spontaneously broken phase is obtained for

$$
\cosh (\mu)>1+\frac{m^{2}}{2}
$$

In the continuum limit the above equation takes the simpler form $\mu>m$.

For concreteness we concentrate in the rest of the paper on $d=1$. In this case the first term in the second line of (1.3) is absent. Assuming periodic boundary conditions and $L$ lattice points one can write

$$
\begin{aligned}
S=\frac{1}{\lambda \alpha^{2}} \sum_{k=1}^{L}( & \frac{u_{k}^{2}+v_{k}^{2}}{2}+\frac{\left(u_{k}^{2}+v_{k}^{2}\right)^{2}}{4} \\
& \left.-\alpha\left(\cosh (\mu)\left(u_{k} u_{k+1}+v_{k} v_{k+1}\right)+i \sinh (\mu)\left(v_{k} u_{k+1}-u_{k} v_{k+1}\right)\right)\right) .
\end{aligned}
$$

As long as $\mu$ is small enough, taking the integration contour to be the product of the real $u_{k}$ and $v_{k}$ axes would not lead to a significant phase factor. As $\mu$ is increased the phase variations along this integration contour become larger and larger and the sign problem appears. To illustrate this (well known) situation we attempted to simulate the system with the standard integration contour on lattices of size $16,24,32, \ldots, 72$. For simplicity we set, as we do for all the simulations in this paper, $\lambda=m=\mu=1$ and estimated the number of configurations that should be sampled in order to obtain a standard error of $\langle S\rangle$ that would be about $2 \%$ of $\langle S\rangle$. Since this is only for an illustrative purpose we were content with identifying a number of configurations that leads to a relative error in the range of $1.6 \%-$ $2.4 \%$. We find that while for 16 and 24 lattice points we need roughly the same number of configurations, for larger lattices the required number of configurations grows fast. We performed a least squares fit for the logarithm of the number of configurations as a function of lattice size for the range $L=24 \ldots 72$ and found that the number of configurations needed grows as $e^{0.21 L}$. We plot the number of configurations needed together with the fit found in figure 1. Assuming this exponential behaviour continues, we can estimate that the running time (on a standard laptop) with no contour modification needed for the stated precision 


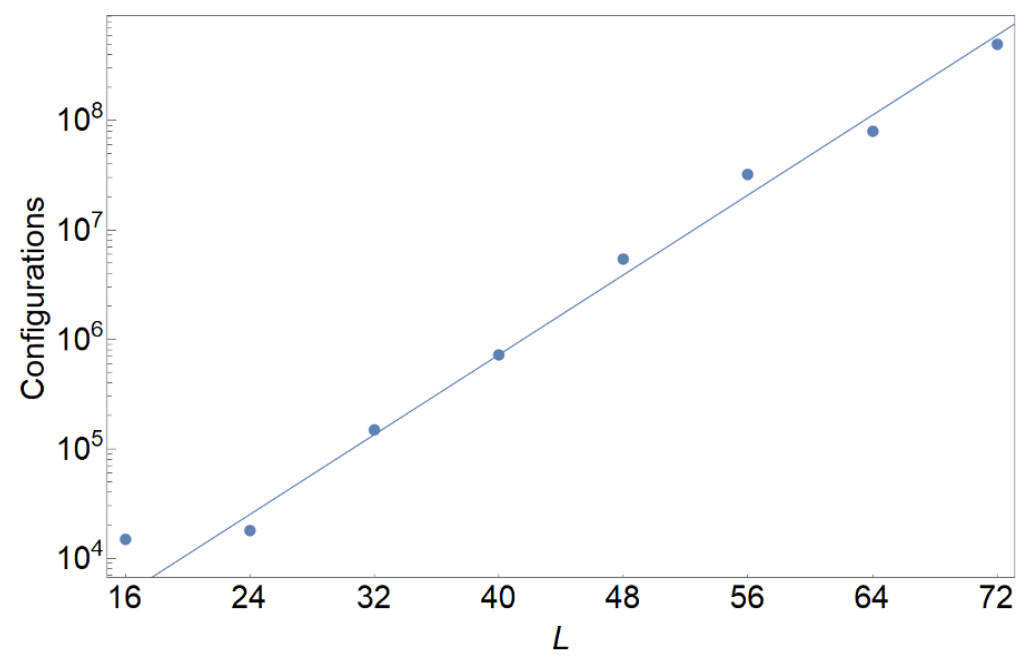

Figure 1. The number of configurations needed for retaining a constant relative precision as a function of lattice size for an unmodified contour grows exponentially as $e^{0.21 L}$. The sign problem is evident.

for $L=96$ would already be of the order of a couple of months. This is significantly longer than the running time with contour deformations studied below, for which the precision is also much better.

In light of the above we look for adequate contour deformations. We write the complexified fields as

$$
u_{k}=x_{k}+i y_{k}, \quad v_{k}=\xi_{k}+i \zeta_{k},
$$

and look for a parametrisation of the contour of the form

$$
y_{k}=y_{k}\left(\left\{x_{m}, \xi_{m}\right\}\right), \quad \zeta_{k}=\zeta_{k}\left(\left\{x_{m}, \xi_{m}\right\}\right),
$$

that is, we intend to use the real parts of the variables as the space over which we integrate. This is not the most general form of a deformed contour: we do not allow contours that return to previous values of $\left\{x_{m}, \xi_{m}\right\}$. However, as we already stressed, there is very large freedom in choosing the integration contour and our choice of parametrisation does not limit us. Finding an integration contour amounts to solving a single equation

$$
\operatorname{Im}(S)=0
$$

in $2 L$ variables. One could get to the conclusion that this is a trivial task. However, the solution must be well defined and free from singularities in the whole space spanned by the $x_{k}$ and $\xi_{k}$, since otherwise the "contour" would not be continuous and Cauchy's theorem would not hold. Moreover, as stated above, care must be taken to ensure that the asymptotic behaviour of the contour is the desired one. While it is still possible to allow the contour to approach other asymptotic regions before returning and heading to the correct one, this should only be allowed as long as $e^{-S}$ is absolutely integrable along such paths. 
How should one choose an integration contour? We suggest to follow an old and important principle of physics: choose a contour that is as simple as possible but not simpler than that. We should now decide upon our criteria for "simplicity" and upon the general considerations that should guide us in our search after good integration contours:

1. Explicit and functionally simple form. We would like to have expressions that can easily be implemented and whose computational cost is small.

2. Approximate expressions. The imaginary part of the action does not have to vanish exactly as in (1.9). A small variation of the phase that does not lead to a severe sign problem can well be tolerated. When possible, we can trade the accuracy of (1.9) for simplicity of the expressions.

3. Include the Jacobian if needed. In order to prevent the residual sign problem, the contribution to the phase coming from the Jacobian can be included $a b$ initio. If the residual phase is small we can follow the previous principle and ignore it, if it simplifies the expressions.

4. Fixed functional form. The functions used for defining the contour (1.8) should have the same (relative) functional dependence on the $x_{k}, \xi_{k}$, for all the $y_{k}, \zeta_{k}$. Here, "relative" means that the dependence of, say, $y_{1}$ on, say, $\xi_{2}$ would be the same as that of $y_{3}$ on $\xi_{4}$. The motivation behind this restriction in not so much the deep physical principle of homogeneity of space-time as the ease of implementation. In particular, since the lattice size can vary, we would like to have a prescription for the deformation that does not change as the lattice size changes.

5. Locality. We would look for contours for which $y_{k}, \zeta_{k}$ could depend on $x_{k}, \xi_{k}$ and maybe on their nearest neighbours. Again, the main motivation is computational rather than the deep physical principle of locality: the evaluation of Jacobians can become significantly cheaper computationally for local expressions. Also, the parallelisation of local expressions is much easier and much more efficient. Moreover, for contours that are defined in terms of some free parameters, locality allows us to fix their values on a small lattice and use the same values for larger lattices. Of course, the last issues is related to the fact that the original action is indeed local. Physical locality, which in the case of (1.1) manifests itself as the restriction that interactions are nearest-neighbour ones, also implies that a contour for which $y_{k}, \zeta_{k}$ only depend on nearest neighbours could grasp the essential contributions to the sign problem.

6. Symmetry. The theory we consider here enjoys a U(1) symmetry. It is not clear a priori that the best possible contours should respect this symmetry. After all, the theory has a spontaneously broken phase. However, when examining ansätze for the form of the contour, one could examine whether the symmetry is respected for ansätze with a small number of parameters. If this is the case, it could be more efficient to restrict the number of parameters at higher levels in a way that respects the symmetry. 
We would like to stress that our approach is practical and that the proposed criteria should be thought of as rules of thumb, to be implemented only as long as it is beneficial. In the current paper we see that we can benefit from a mild breaking of translational invariance for our choice of contours. ${ }^{1}$ This goes against the spirit of points 4 and 6 above, but it turns out that since this breaking is mild the expressions still enjoy the benefits presented above while another important task (a fast calculation of the Jacobian) is also accomplished. As another example of breaking these rules of thumb consider a theory with fermions that leads to a bosonic action, which is not local due to the presence of a fermionic determinant, whose computational cost is high. It is clear that in such a case one does not have to insist neither on local expressions, since they would not be able to account for the actual source of the sign problem, nor on a very fast algorithm for evaluating the Jacobian, since the complexity of the simulation would anyway be restricted by the evaluation of the fermionic determinant.

The rest of the paper is organised as follows: in section 2 we note that the leading source for the sign problem in the current case comes from nearest neighbour interactions. We then propose to expand the functions (1.8) with respect to the small parameter $\alpha$ in order to eliminate this leading contribution to the imaginary part of the action, i.e. we identify functions (1.8) that solve (1.9) to lowest order with respect to $\alpha$. Then, in section 3 , we generalise these functions to an ansatz that depends on some free parameters. Next, in section 4, we study the Jacobians related to the contours defined by our ansatz. Simulation results are presented in section 5 and we end with some discussion in section 6 .

Note added. While this work was prepared the papers [24-26] appeared, in which some similar ideas were presented. Our approach shares with [26] the low computational complexity and the ability to use a relatively small number of parameters, while generalising the ansatz for the contour in a way that enables taking into account the interaction between nearby lattice points.

\section{Simple integration contours}

In order to find an approximation for the contour we write the imaginary part of the action (1.6) in terms of the unknown functions $y_{k}\left(\left\{x_{m}, \xi_{m}\right\}\right)$ and $\zeta_{k}\left(\left\{x_{m}, \xi_{m}\right\}\right)$. If we were to choose

$$
y_{k}=\zeta_{k}=0
$$

we would have obtained

$$
\operatorname{Im}(S)=\frac{\sinh (\mu)}{\lambda \alpha} \sum_{k=1}^{L}\left(x_{k} \xi_{k+1}-x_{k+1} \xi_{k}\right) .
$$

In the following, we refer to the undeformed contour defined by the functions (2.1) as "contour 0".

\footnotetext{
${ }^{1}$ This does not imply of course that we break the physical translational invariance, since the integration result does not depend on the choice of the contour and is therefore not more physical than a gauge choice that breaks a symmetry.
} 
In order to obtain better behaved contours, we expand the imaginary part of the action in powers of $\alpha$. Recall that the definition of $\alpha$ (1.4) implies that it is bounded from above by $\frac{1}{2 d}=\frac{1}{2}$ and that the $\alpha \rightarrow 0$ limit, around which we expand, is the infinite mass limit. We assume that the functions we are after can also be expanded this way and we set their zero order term to zero, that is we write

$$
y_{k}=\sum_{j=1}^{\infty} \alpha^{j} y_{k}^{(j)} .
$$

In the current paper we will only be interested in the first term in this expansion and in generalisations of its functional form. We now write

$$
y_{k}^{(1)}=\sigma \tilde{y}_{k}, \quad \zeta_{k}^{(1)}=\sigma \tilde{\zeta}_{k},
$$

where we defined

$$
\sigma \equiv \alpha \sinh (\mu)
$$

At the lowest order in the $\alpha$ expansion the imaginary part of the action takes the form,

$$
\operatorname{Im}(S)=\frac{\sinh (\mu)}{\lambda \alpha} \sum_{k}\left(\left(x_{k} \xi_{k+1}-x_{k+1} \xi_{k}\right)+\left(x_{k}\left(1+x_{k}^{2}+\xi_{k}^{2}\right)\right) \tilde{y}_{k}+\left(\xi_{k}\left(1+x_{k}^{2}+\xi_{k}^{2}\right)\right) \tilde{\zeta}_{k}\right) .
$$

Setting this expression to zero gives a single linear equation in the $2 L$ unknowns $\tilde{y}_{k}$ and $\tilde{\zeta}_{k}$. Such an equation has many solutions. However, we are interested only in solutions that are continuous with proper asymptotic behaviour and for which the dependence of $y_{k}$ and $\zeta_{k}$ on $x_{k}, x_{k+1}, \xi_{k}, \xi_{k+1}$ is the same for all $k$.

We can look for a solution in which the $\tilde{y}_{k}$ term cancels the contributions of $x_{k} \xi_{k+1}$ and the $\tilde{\zeta}_{k}$ term cancels the contributions of $-x_{k+1} \xi_{k}$ for all $k$ even before summation. Thus, we set,

$$
\begin{gathered}
\tilde{y}_{k}=-\frac{\xi_{k+1}}{1+x_{k}^{2}+\xi_{k}^{2}}, \\
\tilde{\zeta}_{k}=\frac{x_{k+1}}{1+x_{k}^{2}+\xi_{k}^{2}} .
\end{gathered}
$$

We refer to the contour defined by (2.7) as "contour 1". Note that this contour respects the $\mathrm{U}(1)$ symmetry of the theory: if we define

$$
\phi_{k} \equiv x_{k}+i \xi_{k}, \quad \psi_{k} \equiv y_{k}+i \zeta_{k}
$$

we can write $(2.7)$ as

$$
\psi_{k}=\frac{i \sigma \phi_{k+1}}{1+\left|\phi_{k}\right|^{2}} .
$$

The U(1) symmetry rotates both $\phi_{k}$ and $\psi_{k}$ in the standard manner.

Nonetheless, contour 1 does break a symmetry. Sending $k+1 \rightarrow k-1$ while either taking a complex conjugation or sending $\mu \rightarrow-\mu$, leaves the action (1.6) invariant. This 
discrete symmetry is broken by the contour. One could act with this symmetry on the contour obtaining an equivalent one defined by,

$$
\psi_{k}=-\frac{i \sigma \phi_{k-1}}{1+\left|\phi_{k}\right|^{2}} .
$$

This contour could have also been obtained by a rewriting of the terms in the sum before deciding that they should vanish even before summation. However, since this contour is equivalent to contour 1 , we do not study it separately.

The expressions (2.7) are not bounded in the limits $\xi_{k+1} \rightarrow \infty$ and $x_{k+1} \rightarrow \infty$ respectively, with all other variables fixed. This means that the asymptotic range covered by them might include also parts of the complexified space unrelated to the original contour. Nonetheless, since these expressions can be obtained by continuously deforming the original integration contour, they would lead to the correct results, as long as they do not pass asymptotic regions over which the integral diverges. One could further worry that even if the integrals along these parts of the contour are finite, they would include large contributions that are supposed to cancel each other. The main contribution to the different phase in this case would come from the Jacobian. Such a scenario could lead to a global sign problem reminiscent of problems with integration along Lefschetz thimbles. For similar reasons integration along a generalised thimble can behave better than integration along the thimble itself $[27,28]$. This problem could be addressed by slightly modifying the contour in order to obtain expressions that are everywhere bounded. A simple possibility is to use the following expressions,

$$
\begin{gathered}
\tilde{y}_{k}=-\frac{\xi_{k+1}}{1+x_{k}^{2}+\xi_{k}^{2}+\xi_{k+1}^{2}}, \\
\tilde{\zeta}_{k}=\frac{x_{k+1}}{1+x_{k}^{2}+\xi_{k}^{2}+x_{k+1}^{2}} .
\end{gathered}
$$

These expressions neither respect the $\mathrm{U}(1)$ symmetry nor do they lead to $\operatorname{Im}(S)=0$ even at the lowest order of our expansion. Instead, they respect a $\mathbb{Z}_{4}$ subgroup of $U(1)$, generated by

$$
x_{k} \rightarrow \xi_{k}, \quad \xi_{k} \rightarrow-x_{k},
$$

while the imaginary part of the action is given by,

$$
\operatorname{Im}(S)=\frac{\sinh (\mu)}{\lambda \alpha} \sum_{k}\left(\frac{x_{k} \xi_{k+1}^{3}}{1+x_{k}^{2}+\xi_{k}^{2}+\xi_{k+1}^{2}}-\frac{\xi_{k} x_{k+1}^{3}}{1+x_{k}^{2}+\xi_{k}^{2}+x_{k+1}^{2}}\right) .
$$

However, it might happen that the benefit from avoiding infinity is more significant for taming the sign problem than this small mismatch. We refer to the contour defined by (2.11) as "contour 2".

Of course, in order to turn (2.7) into bounded expressions, one could also use variants of (2.11). In particular, one could extrapolate between (2.7) and (2.11) by introducing a constant $\kappa$ and setting

$$
\begin{gathered}
\tilde{y}_{k}=-\frac{\xi_{k+1}}{1+x_{k}^{2}+\xi_{k}^{2}+\kappa \xi_{k+1}^{2}}, \\
\tilde{\zeta}_{k}=\frac{x_{k+1}}{1+x_{k}^{2}+\xi_{k}^{2}+\kappa x_{k+1}^{2}} .
\end{gathered}
$$


Now, for $\kappa=0$ we obtain (2.7), while (2.11) is obtained for $\kappa=1$ and values of $\kappa>1$ can also be considered. We could try to look for the value of $\kappa$ for which the sign problem is most significantly reduced. Eq. (2.14) is an ansatz for the contour depending on a single parameter. We refer to this 1-parameter ansatz with an optimal choice of $\kappa$ as "contour 3". We now turn to define a more general ansatz.

\section{A general ansatz for the integration contour}

In order to obtain better behaved contours, it might be useful to generalise the expressions considered so far. A generalised contour could account for approximations we have made, as well as for the phase factor due to the Jacobian, that we did not considered in the discussion so far. A natural generalisation would be to rely on the functional forms defining the contours proposed above, while changing the powers of the various factors and allowing for a somewhat less local functional dependence. One can see that if we were to go to higher orders in our $\alpha$ expansion, such terms would have indeed appeared. We therefore consider the following functional form for defining the contour, ${ }^{2}$

$$
\begin{aligned}
& \tilde{y}_{k}=-\frac{\sum_{\mu \nu \rho \sigma \tau \phi} a_{\mu \nu \rho \sigma \tau \phi} x_{k}^{\mu} x_{k-1}^{\nu} x_{k+1}^{\rho} \xi_{k}^{\sigma} \xi_{k-1}^{\tau} \xi_{k+1}^{\phi}}{1+\sum_{\mu \nu \rho \sigma \tau \phi} b_{\mu \nu \rho \sigma \tau \phi} x_{k}^{\mu} x_{k-1}^{\nu} x_{k+1}^{\rho} \xi_{k}^{\sigma} \xi_{k-1}^{\tau} \xi_{k+1}^{\phi}}, \\
& \tilde{\zeta}_{k}=\frac{\sum_{\mu \nu \rho \sigma \tau \phi} a_{\mu \nu \rho \sigma \tau \phi} x_{k}^{\sigma} x_{k-1}^{\tau} x_{k+1}^{\phi} \xi_{k}^{\mu} \xi_{k-1}^{\nu} \xi_{k+1}^{\rho}}{1+\sum_{\mu \nu \rho \sigma \tau \phi} b_{\mu \nu \rho \sigma \tau \phi} x_{k}^{\sigma} x_{k-1}^{\tau} x_{k+1}^{\phi} \xi_{k}^{\mu} \xi_{k-1}^{\nu} \xi_{k+1}^{\rho}} .
\end{aligned}
$$

Here the sum in the numerator runs over all non-negative integers $\mu, \nu, \rho, \sigma, \tau, \phi$ such that $0 \leq \mu+\nu+\rho+\sigma+\tau+\phi \leq p$, and the sum in denominator runs over all even non-negative integers $\mu, \nu, \rho, \sigma, \tau, \phi$ such that $2 \leq \mu+\nu+\rho+\sigma+\tau+\phi \leq q$. We define $(p, q)$ to be the order of the contour. The reason for restricting the sum to only even integers in the denominator is to ensure that the denominator is always positive so that the contour always remains continuous for finite values of the integration variable. This also requires that the $b_{\mu \nu \rho \sigma \tau \phi}$ are non-negative. The requirement of obtaining proper boundary conditions implies the inequality $q \geq p$. One can further restrict the ansatz by requiring that no terms appear in the numerator with powers of any variable that does not appear in the denominator with at least the same power. This will result in contours that are everywhere bounded.

The ansatz (3.1) includes all contours considered so far and generalises them using rational functions. One advantage of using rational function as in (3.1) is that they are easy to differentiate and so the Jacobian can be explicitly written. The ansatz also has a sign choice that reminds of the previously considered contours. Specifically, if one only considers non-zero coefficients in the numerator for even values of $\mu+\nu+\rho$ and odd values of $\sigma+\tau+\phi$, the $\mathbb{Z}_{4}$ symmetry (2.12) is respected. A further possible restriction is to impose the whole $\mathrm{U}(1)$ symmetry, that is to consider only expressions that can be written in a way that generalises (2.9). Such a restriction would not necessarily lead to the best contours. However, if it turns out that the U(1) symmetry gives approximately the best

\footnotetext{
${ }^{2}$ Note that $\mu, \nu, \rho, \sigma, \tau, \phi$ are powers to which the variables are raised, not indices, which would have made no sense in the current one-dimensional case.
} 
contours at low contour order, it would make sense to simplify the optimisation procedure by imposing the $\mathrm{U}(1)$ symmetry on the ansatz ab initio, reducing in this way the amount of free parameters needed to be optimised.

It is easy to include higher order terms in this contour simply by increasing the order. Similarly, longer-range terms could be included by adding to the ansatz expressions depending on $x_{k \pm 2}, \xi_{k \pm 2}$ etc. However, this comes at a cost; we need to determine the parameters $a_{\mu \nu \rho \sigma \tau \phi}, b_{\mu \nu \rho \sigma \tau \phi}$. There may be a large number of these; without imposing restrictions on the coefficients, already at order $(2,2)$ there are 34 free parameters. Furthermore, the optimal contour will presumably depend on the chemical potential $\mu$, the interaction strength $\lambda$, and on $\alpha$.

Another important restriction is to contours for which the Jacobian can be efficiently evaluated. We discuss this issue in the next section. For now we just mention that this restriction implies that one should consider in (3.1) either only terms with $x_{k+1}$ and $\xi_{k+1}$ or terms with $x_{k-1}$ and $\xi_{k-1}$, but not both. We refer in what follows to the use of only $x_{k+1}$ and $\xi_{k+1}$ as "forward nearest neighbour" and to a forward nearest neighbour ansatz with optimised parameters as "contour 4". An optimised choice for ansatz parameters without this restriction would be referred to as "contour 5".

Choosing the best contour sounds like a daunting task; on a large lattice where the sign problem is severe we would expect that for generic contour parameters $a_{\mu \nu \rho \sigma \tau \phi}, b_{\mu \nu \rho \sigma \tau \phi}$ the mean phase factor will be so small that it will be statistically indistinguishable from zero. How can we then decide how to change the parameters to obtain a larger mean phase factor? The solution is to tune the contour on small lattices. Since the action is local, the imaginary part of the action is presumably mostly due to local contributions rather than long-range correlations. Our ansatz is also local. Hence, if we tune the parameters $a_{\mu \nu \rho \sigma \tau \phi}, b_{\mu \nu \rho \sigma \tau \phi}$ to maximise the mean phase factor on a small volume we will obtain a contour that minimises these contributions. Then, if we use a contour defined by the same parameters on a larger volume the imaginary part of the action should still be small. If needed, one could start on a large lattice with the parameters fixed on a small one and update them, but locality implies that generically this would not lead to a significant improvement. We explain the methods used for optimising the ansatz parameters in subsection 5.1.

\section{The Jacobian}

To do calculations with these contours we must parametrise $u_{k}$ and $v_{k}$ in some convenient way. The most natural thing to do is to use the real parts $x_{k}$ and $\xi_{k}$. We must then include the Jacobian determinant in the evaluation,

$$
\operatorname{det}(J)=\operatorname{det}\left(\begin{array}{ccccc}
\frac{\partial u_{1}}{\partial x_{1}} & \frac{\partial u_{1}}{\partial \xi_{1}} & \frac{\partial u_{1}}{\partial x_{2}} & \frac{\partial u_{1}}{\partial \xi_{2}} & \ldots \\
\frac{\partial v_{1}}{\partial x_{1}} & \frac{\partial v_{1}}{\partial \xi_{1}} & \frac{\partial v_{1}}{\partial x_{2}} & \frac{\partial v_{1}}{\partial \xi_{2}} & \ldots \\
\frac{\partial u_{2}}{\partial x_{1}} & \frac{\partial u_{2}}{\partial \xi_{1}} & \frac{\partial u_{2}}{\partial x_{2}} & \frac{\partial u_{2}}{\partial \xi_{2}} & \ldots \\
\frac{\partial v_{2}}{\partial x_{1}} & \frac{\partial v_{2}}{\partial \xi_{1}} & \frac{\partial v_{2}}{\partial x_{2}} & \frac{\partial v_{2}}{\partial \xi_{2}} & \ldots \\
\vdots & \vdots & \vdots & \vdots & \ddots
\end{array}\right)
$$


For contour 1 (2.7) the Jacobian matrix takes the form,

$$
J=\left(\begin{array}{ccccccc}
1+2 i \sigma \frac{x_{1} \xi_{2}}{d_{1}^{2}} & 2 i \sigma \frac{\xi_{1} \xi_{2}}{d_{1}^{2}} & 0 & -i \sigma \frac{1}{d_{1}} & 0 & 0 & 0 \ldots \\
-2 i \sigma \frac{x_{1} x_{2}}{d_{1}^{2}} & 1-2 i \sigma \frac{\xi_{1} x_{2}}{d_{1}^{2}} & i \sigma \frac{1}{d_{1}} & 0 & 0 & 0 & 0 \ldots \\
0 & 0 & 1+2 i \sigma \frac{x_{2} \xi_{3}}{d_{2}^{2}} & 2 i \sigma \frac{\xi_{2} \xi_{3}}{d_{2}^{2}} & 0 & -i \sigma \frac{1}{d_{2}} & 0 \ldots \\
0 & 0 & -2 i \sigma \frac{x_{2} x_{3}}{d_{2}^{2}} & 1-2 i \sigma \frac{\xi_{2} x_{3}}{d_{2}^{2}} & i \sigma \frac{1}{d_{2}} & 0 & 0 \ldots \\
\vdots & \vdots & & & \ddots & \ddots & \\
0 & -i \sigma \frac{1}{d_{L}} & 0 & 0 & \ldots & & \\
i \sigma \frac{1}{d_{L}} & 0 & 0 & 0 & \ldots & &
\end{array}\right)
$$

where we defined

$$
d_{k} \equiv 1+x_{k}^{2}+\xi_{k}^{2} .
$$

For contour 2 (2.11) we have a slightly more complicated Jacobian matrix,

$$
J=\left(\begin{array}{ccccccc}
1+2 i \sigma \frac{x_{1} \xi_{2}}{\tilde{d}_{1}^{2}} & 2 i \sigma \frac{\xi_{1} \xi_{2}}{\tilde{d}_{1}^{2}} & 0 & -i \sigma \frac{\tilde{d}_{1}-2 \xi_{2}^{2}}{\tilde{d}_{1}^{2}} & 0 & 0 & 0 \ldots \\
-2 i \sigma \frac{x_{1} x_{2}}{\hat{d}_{1}^{2}} & 1-2 i \sigma \frac{\xi_{1} x_{2}}{\hat{d}_{1}^{2}} & i \sigma \frac{\hat{d}_{1}-2 x_{2}^{2}}{\hat{d}_{1}^{2}} & 0 & 0 & 0 & 0 \ldots \\
0 & 0 & 1+2 i \sigma \frac{x_{2} \xi_{3}}{\tilde{d}_{2}^{2}} & 2 i \sigma \frac{\xi_{2} \xi_{3}}{\tilde{d}_{2}^{2}} & 0 & -i \sigma \frac{\tilde{d}_{2}-2 \xi_{3}^{2}}{\tilde{d}_{2}^{2}} & 0 \ldots \\
0 & 0 & -2 i \sigma \frac{x_{2} x_{3}}{\hat{d}_{2}^{2}} & 1-2 i \sigma \frac{\xi_{2} x_{3}}{\hat{d}_{2}^{2}} & i \sigma \frac{\hat{d}_{2}-2 x_{3}^{2}}{\hat{d}_{2}^{2}} & 0 & 0 \ldots \\
\vdots & \vdots & & 0 & \ddots & \ddots & \\
0 & -i \sigma \frac{\tilde{d}_{L}-2 \xi_{1}^{2}}{\hat{d}_{L}^{2}} & 0 & 0 & \ldots & & \\
i \sigma \frac{\hat{d}_{L}-2 x_{1}^{2}}{\hat{d}_{L}^{2}} & 0 & 0 & 0 & \ldots & &
\end{array}\right)
$$

where now we defined

$$
\tilde{d}_{k}=1+x_{k}^{2}+\xi_{k}^{2}+\xi_{k+1}^{2}, \quad \hat{d}_{k}=1+x_{k}^{2}+\xi_{k}^{2}+x_{k+1}^{2} .
$$

Similarly it is straightforward to derive the Jacobian for our general ansatz (3.1).

We note, that both (4.2) and (4.4) are almost upper-block-bidiagonal with $2 \times 2$ blocks. The block structure comes from the fact that we have two fields in every lattice site and the upper-bidiagonal structure originates from the use of forward nearest neighbour in the definition of the contours. Thus, in the general forward nearest neighbour case, the Jacobian matrix takes the form

$$
J=\left(\begin{array}{ccccc}
A_{1} & B_{1} & 0 & 0 & \ldots \\
0 & A_{2} & B_{2} & 0 & \ldots \\
\vdots & \ddots & \ddots & \ddots & 0 \\
0 & \ldots & 0 & A_{L-1} & B_{L-1} \\
B_{L} & 0 & \ldots & 0 & A_{L}
\end{array}\right)
$$

The only term that does not fit the upper-bidiagonal form is $B_{L}$ (marked in red). This term is there in light of the periodic boundary conditions. Had this term been absent, the 
Jacobian determinant would be the product of $2 \times 2$ determinants. However, it is easy to evaluate the Jacobian determinant even when this term is present. If the blocks were just numbers we could have used elementary row operations in order to eliminate $B_{L-1}$, then eliminate $B_{L-2}$, and so on, obtaining a matrix whose determinant is given by

$$
\operatorname{det}(J)=\left(A_{1}-(-1)^{L} B_{1} A_{2}^{-1} \cdot \ldots \cdot B_{L-1} A_{L}^{-1} B_{L}\right) \cdot A_{2} \cdot \ldots \cdot A_{L} .
$$

In the current case where we deal with blocks instead of numbers, we can perform "elementary row operations" by left multiplication by "elementary block matrices", whose determinant is unity. This leads to a very similar expression,

$$
\begin{aligned}
\operatorname{det}(J) & =\operatorname{det}\left(A_{1}-(-1)^{L} B_{1} A_{2}^{-1} \cdot \ldots \cdot B_{L-1} A_{L}^{-1} B_{L}\right) \cdot \operatorname{det}\left(A_{2}\right) \cdot \ldots \cdot \operatorname{det}\left(A_{L}\right) \\
& =\operatorname{det}\left(\mathbf{1}-(-1)^{L} S_{1} S_{2} \cdot \ldots \cdot S_{L}\right) \cdot \operatorname{det}\left(A_{1}\right) \cdot \ldots \cdot \operatorname{det}\left(A_{L}\right),
\end{aligned}
$$

where we defined

$$
S_{k} \equiv A_{k}^{-1} B_{k} .
$$

We reduced the evaluation of the determinant to the evaluation of a product of block matrices. Thus we resolved the complexity related to the evaluation of the determinant. One could still worry that since the determinant includes a product of $L+1$ terms of which the evaluation of the first involves a product of $L$ matrices it would lead to a complexity of $O\left(L^{2}\right)$. This is not the case. The locality of our prescription implies that when we change $x_{k}$ or $\xi_{k}$ we should only update $A_{k-1}, A_{k}, B_{k-1}, B_{k}$. Dividing the product by the previous value of $\operatorname{det}\left(A_{k-1}\right) \operatorname{det}\left(A_{k}\right)$ and multiplying by the new value of this factor is of $O(1)$. As for the first term in the product, symmetry of the problem implies that it can be written in any cyclic order,

$$
\operatorname{det}\left(\mathbf{1}-(-1)^{L} S_{1} S_{2} \cdot \ldots \cdot S_{L-1} S_{L}\right)=\operatorname{det}\left(\mathbf{1}-(-1)^{L} S_{k} S_{k+1} \cdot \ldots \cdot S_{k-2} S_{k-1}\right) .
$$

This identity can also be proven explicitly, for example by expressing the determinant in terms of a trace and utilising the cyclicity property of the trace. Thus, when we initiate the simulation we define

$$
M \equiv S_{L} S_{1} \cdot \ldots \cdot S_{L-1}
$$

We start by updating $x_{1}$ after which we change

$$
M \rightarrow\left(S_{L} S_{1}\right)_{\text {new }}\left(S_{L} S_{1}\right)_{\mathrm{old}}^{-1} M .
$$

We then update $\xi_{1}$ after which we change, ${ }^{3}$

$$
M \rightarrow\left(S_{1}\right)_{\text {new }}\left(S_{L} S_{1}\right)_{\text {old }}^{-1} M\left(S_{L}\right)_{\text {new }} .
$$

We used the cyclicity property (4.10) in order to obtain an expression that is adequate for $x_{2}$. We then continue with $x_{2}, \xi_{2}$ and so on. All the manipulations described are of $O(1)$ and so do not increase the complexity.

\footnotetext{
${ }^{3}$ In fact, we update $x_{k}$ and $\xi_{k}$ at a single step, so we only have to use the second update. Note, that one must perform the second update even when the update for $x_{k}, \xi_{k}$ is rejected, since it brings the matrix $M$ to the form needed for updating the next site.
} 
The above algorithm might not always work: while the $A_{k}$ are always invertible the $B_{k}$ can be non-invertible for specific contours. Moreover, even when the $B_{k}$ are invertible, they might be close to being singular. This, together with the fact that these are complex matrices, could lead to stability issues for the algorithm. There are several options for handling this obstacle:

1. One could use a slow algorithm to evaluate the determinant without using the nearlybidiagonal structure, such as LU decomposition $\left(O\left(L^{3}\right)\right.$ per site so $O\left(L^{4}\right)$ per sweep), or evaluate $M$ using its definition (4.11) after every update, which leads to complexity of $O\left(L^{2}\right)$. This solution is, of course, not the desired one, since even the $O\left(L^{2}\right)$ would be much more restrictive than an $O(L)$ algorithm.

2. The problem described would emerge only for specific values of the parameters defining the general contour. One could then choose a different set of parameters, for which the $B_{k}$ are generically large and the problem does not occur.

3. It is possible to deform all the contours, but one. Suppose we decide not to deform $u_{1}, v_{1}$, this modifies the expression for the Jacobian to the simpler form,

$$
\operatorname{det}(J)=\operatorname{det}\left(A_{2}\right) \cdot \ldots \cdot \operatorname{det}\left(A_{L}\right) .
$$

While such a choice would certainly decrease the mean phase factor, we expect that this change would be $L$-independent and so would not change by much the range of validity of the given contour, i.e. the unmodified and modified contours should begin to suffer from the sign problem at not too different values of $L$. If this option is chosen one should verify that observables at $k=1$ do not behave in any anomalous way. While analytically this is obvious, the asymmetric treatment of a single point can in principle induce different numerical errors in this point as compared to the other ones.

4. Since the numerical instability would usually occur for small values of $B_{k}$, one could expect that for large enough $L$, the matrix $M$ would become extremely small and the approximation

$$
\operatorname{det}(J) \simeq \operatorname{det}\left(A_{1}\right) \cdot \ldots \cdot \operatorname{det}\left(A_{L}\right)
$$

would become exact within the simulation precision. This expression is almost identical to (4.14), but the decrease of the phase factor by a constant is now absent. Moreover, since in this case (as well as in the previous one) there is no need to evaluate the matrix $M$, the computational cost is further reduced.

All these options are examined in subsection 5.3.

The discussion so far is inadequate for the general form for the contour (3.1), without imposing the forward nearest neighbour restriction. In this general case the Jacobian matrix is almost tridiagonal-block-matrix and we have no fast algorithm for evaluating it. In the next section we examine the various contours, including contour 5, for which we use a slow algorithm. It turns out that there is no significant improvement in going 
from contour 4 to contour 5 . The amount of free parameters in contour 4 is much smaller than in contour 5 . Hence, the restriction to forward nearest neighbour contours could even enable one to get to a better resolution of the sign problem given a fixed number of free parameters, since then higher $(p, q)$ values can be considered.

\section{Results}

As mentioned in the introduction, we examine the method presented in this paper by simulating the system with $\lambda=m=\mu=1$. The simulation is performed using a Fortran code. In all simulations we take 300,000 configurations. The jackknife method is used for the evaluation of statistical errors. We examine the observables $\langle S\rangle$ and $\left\langle u_{k}^{2}+v_{k}^{2}\right\rangle$ as a function of lattice size for the different contours suggested. Since we only illustrate the method we do not study other observables. Of course, one could examine in this way also the average density and other observables. The mean phase factor for the same contours is also studied, since it serves as an indicator to the severity of the sign problem. As expected, this factor decreases as the lattice size is increased. We find high correlation between problems with the observables we examine and small values of the mean phase factor. Thus, it is natural to expect that problems related to the sign problem with other observables would begin to emerge roughly for the same lattice size as for the observables studied here.

We simulate all the contours described above: the undeformed contour 0 (2.1), contour 1 (2.7), contour 2 (2.11), contour 3 (2.14), contour 4, which is a forward nearest neighbour version of the general ansatz $(3.1)$ for $(p, q)=(1,2)$, and contour 5 , which is an unrestricted version of the general ansatz $(3.1)$ for $(p, q)=(1,2)$. We use the fast algorithm for the evaluation of the Jacobian presented in section 4 for all contours, except contour 5 , for which it is not applicable.

Contours 3,4,5 are representatives within some given ansätze. In subsection 5.1, we present the approach we use for deciding which values should the parameters take. Next, in subsection 5.2, we present the main results of our simulations. First, we examine all the contours for $L=4,8,16,24,32, \ldots, 96$. Then, we offer a prediction for the onset of the sign problem for the various contours, which we examine using $L=100,150,200,250, \ldots, 1000$. Since contour 5 is evaluated using a slow algorithm, we do not examine this prediction for this contour. As mentioned in section 4, the fast algorithm for evaluating contours $1,2,3,4$ may suffer from numerical instability. We monitor this issue in our simulations. To that end, we evaluated $M$ using its original definition (4.11) at the end of each sweep and compared to the result obtained by successively using the update algorithm (4.13). ${ }^{4}$ While contours 1,2,3 show no numerical instability, for contour 4 the relative size of the components of $M$, evaluated in these two ways, differs from unity by more the one part per million (the threshold we used), already at $L=16$. We examine possible resolutions of the numerical instability in subsection 5.3.

\footnotetext{
${ }^{4}$ Note, that since this check is performed at the end of a sweep, it has a computational cost of $O(L)$ so it does not increase the asymptotic cost.
} 


\subsection{Fitting the parameters}

An important characteristic of our approach is the locality of the contours and of the action, which implies that values of the parameters that minimises the sign problem for some large enough $L$, would also minimises the sign problem for higher values of $L$. This fact enables us to identify the parameters for a given value of $L$, for which the evaluation is fast, and use the same values for all values of $L$. We then verify that small changes of the parameters for larger values of $L$ do not lead to further improvement with dealing with the sign problem. In all these evaluations, we use the mean phase factor as a measure of the severity of the sign problem.

Finding the best fit for defining contour 3 is quite simple, since there is only a single parameter in this case. We examine $L=16$ for different values of $\kappa$ and find that the mean phase factor is roughly constant in the range $0<\kappa<0.5$ taking values in the range $(0.708-0.715)$ and begins to drop as $\kappa$ is further increased. We choose the middle of this range, $\kappa=0.25$ as the representative for defining contour 3 , although slightly larger values for the mean phase factor were obtained both above and below this value. However, the difference is very mild and could well be of statistical nature.

Contours 4 and 5 have several parameters and finding them is less trivial. Again, we use the locality for finding the values of the parameters for a fixed $L$, this time for $L=8$. To that end, optimisation of the contour was performed one parameter at a time, by simulating for several values of the parameter, fitting a quadratic to the mean phases obtained, and updating the parameter to the maximum of this quadratic. More efficient methods, e.g. steepest ascent, could also be used in principle. However, we find that convergence using this method is very rapid, typically within 10 or so iterations, so using more efficient methods is unnecessary. Using this method, we obtain the following values for the parameters defining contour 4 ,

$$
\begin{array}{ll}
a_{000001}=0.56, & a_{000100}=1.88, \\
b_{000002}=0.03, & b_{000200}=0.20, \quad b_{002000}=0.05,
\end{array}
$$

with all other contour parameters equal to zero. Notice that the parameters are very close to a $\mathrm{U}(1)$ symmetric contour, i.e. $b_{000002} \simeq b_{002000}$ and $b_{000200} \simeq b_{200000}$. We use this fact in subsection 5.3 where we look for alternative values for the parameters. There, we preform a search in the space of $U(1)$ symmetric contours, which is defined by only four free parameters. In this search we use a Metropolis-like approach: we choose a point in the space of the four free parameters and run the simulation with these values. We then consider a nearby configuration in this space and decide whether to move to this point or not using the weight function $\frac{1}{1-\left\langle e^{i \operatorname{Im}(S)}\right\rangle_{P Q}}$. Other functions that become large near the optimal mean phase value of unity could also be used as weight functions and again, other methods could also be used. However, this is not needed, since results are easily obtained with the current approach. During this random walk we keep track of values of the parameters that lead to mean phase factors higher than some chosen cutoff value. 
For contour 5 we use again the initial approach used for defining contour 4 and find,

$$
\begin{array}{llll}
a_{000001} & =0.73, & a_{000010}=0.15, & a_{000100}=1.77, \\
b_{000020}=0.03, & b_{000200}=0.27, & b_{002000}=0.05, & b_{200000}=0.28,
\end{array}
$$

with all other contour parameters equal to zero. We see that these values are not too far from the ones of contour 4 and the values that are inconsistent with a forward nearest neighbour contour are small. Thus, we expect that the benefit from using the slow contour 5 over contour 4 is not too high.

One could wonder about the values of the parameters obtained: they define contours that differ quite a lot from contours 1,2,3. In particular the weight of $x_{k}$ and $\xi_{k}$ in the numerators is higher than that of $x_{k+1}$ and $\xi_{k+1}$. What could be the reason for that? We propose the following observation: an exact and simple solution to the equation $\operatorname{Im}(S)=$ 0 exists, which also leads to a constant Jacobian and hence could seem as a complete resolution of the sign problem,

$$
y_{k}=-\xi_{k}, \quad \zeta_{k}=x_{k}
$$

However, not only $\operatorname{Im}(S)=0$ on this contour, but actually $S=0$. Hence, it neither leads to a convergent action nor does it obey the proper boundary conditions and is therefore inadequate as an integration contour. Nonetheless, it might be beneficial to go in the direction of such a contour before changing the course towards the one of contour 1 . It seems to us that contours 4 and 5 give such an interpolation between these two contours.

\subsection{The observables and the mean phase factor for the various contours}

In figure 2 we plot the action density, that is, the expectation value of the action divided by the lattice size, for the various contours described above. The different contours are horizontally separated to enable distinguishing them, as many of them overlap. Since it is hard to infer the standard errors from the plot they are also displayed separately. It is obvious that one cannot trust contour 0 for $L \gtrsim 32$. As for the other contours, they all give the same results, which are consistent with the expectation of obtaining a constant action density for large $L$. Given the deformation of the contours and the fact that the action is complex, we could have obtained general complex results. In fact, all the results are consistent with being real. The largest value for $\frac{|\operatorname{Im}\langle S\rangle|}{L}$ is 0.1 for contour 0 and about 0.004 for contour 3. It is smaller for the other contours. This gives further support to our method.

It seems that there is no significant difference between contours $1,2,3$ as far as the standard error of the action is concerned. Initially it goes down, but then, around $L=40$, it starts to go up again. The situation with contours 4 and 5 is less clear, since the tendencies are masked by noise. It is hard to infer whether they started going up already or not. In general, there can be several reasons for a dependence of the standard error on lattice size: since we are not at a critical point, observables evaluated at sites separated by more than a finite correlation length are roughly independent. We then expect the standard error of the action to scale like that of $L / l_{\text {corr }}$ independent measurements of the same object, that is, like $\sqrt{L}$. Thus, if this was the only relevant effect, we would have 

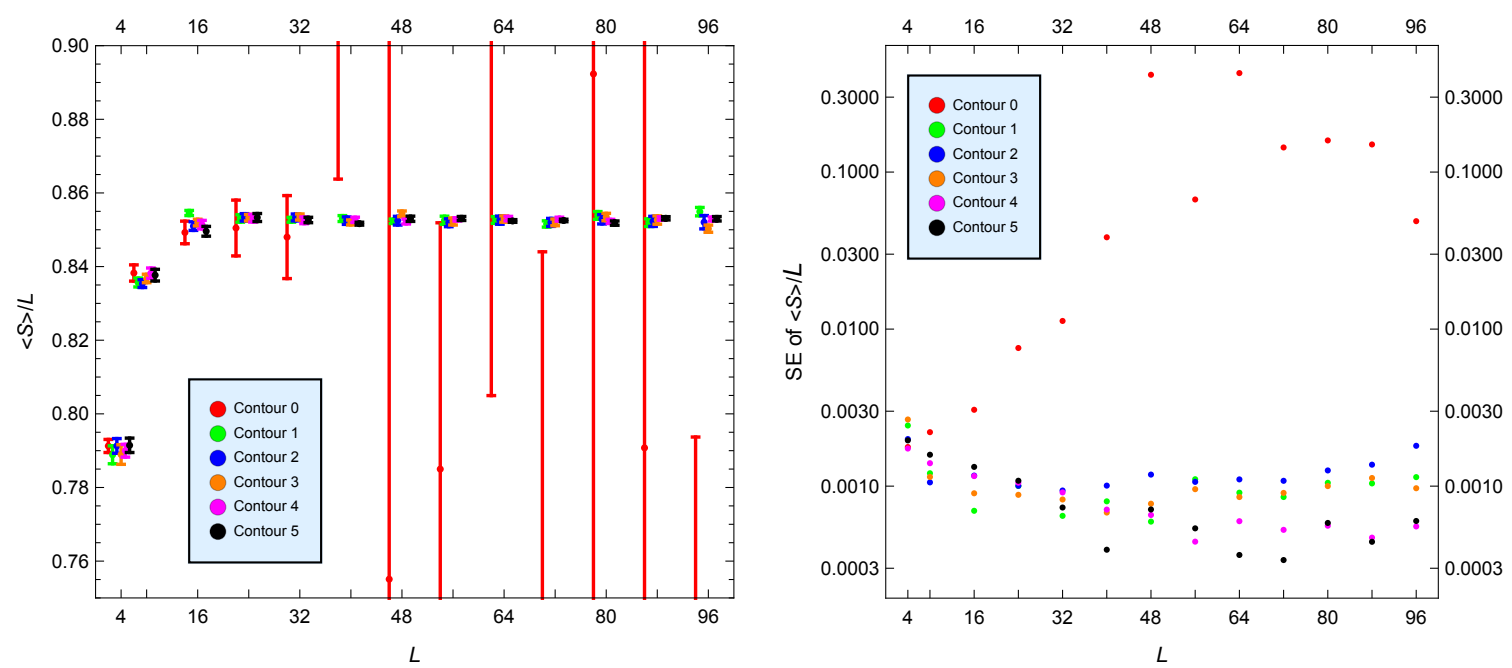

Figure 2. Left: the action density as a function of lattice size. Different contours are separated horizontally for clarity. Right: the standard error of the action density. The various contours are colour marked.
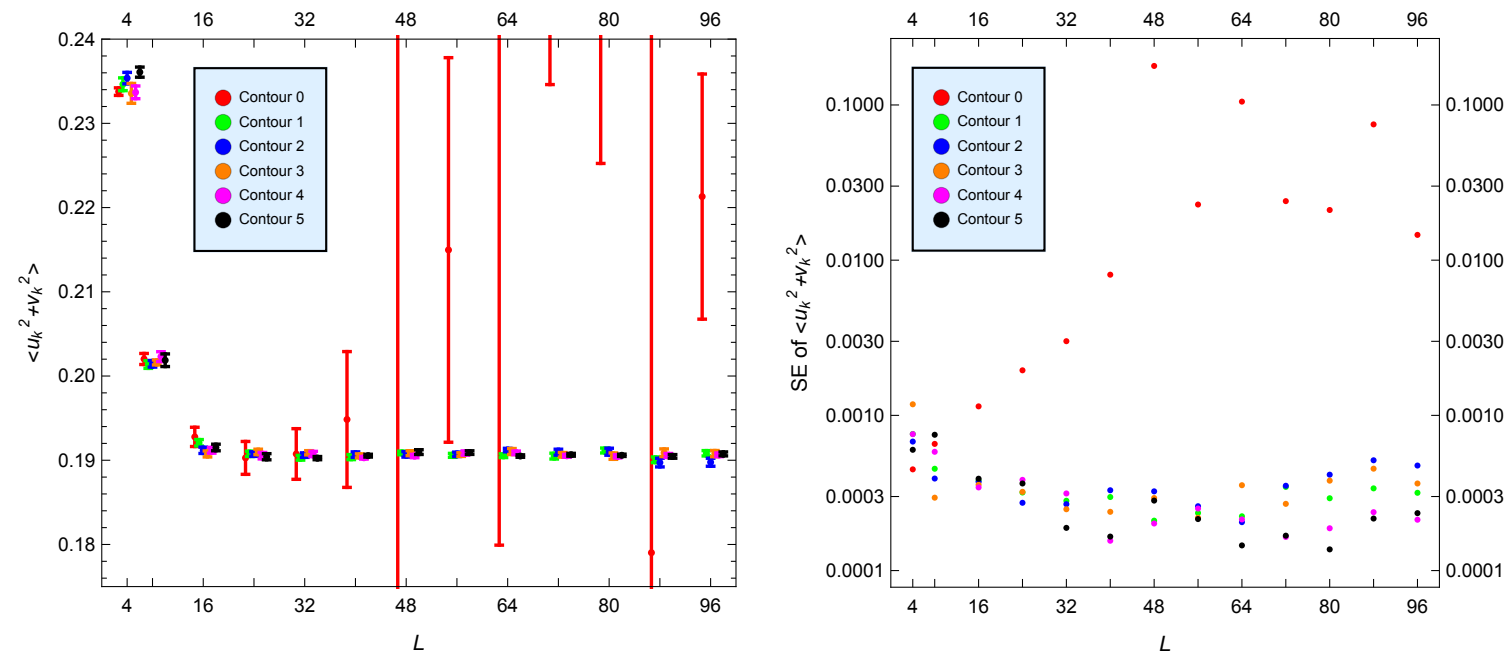

Figure 3. Left: $\left\langle u_{k}^{2}+v_{k}^{2}\right\rangle$ as a function of lattice size. Different contours are separated horizontally for clarity. Right: the standard error of $\left\langle u_{k}^{2}+v_{k}^{2}\right\rangle$. The various contours are colour marked.

expected the standard error of the action density to scale like $L^{-\frac{1}{2}}$. However, there are two effects that increase the size of the standard error: the increase of the autocorrelation time and the sign problem. Thus, one could suspect that contours 1,2,3 are closer to developing a sign problem than contours 4 and 5 , although it is hard to make this statement into a quantitative one based solely on the values of the standard error.

In figure 3 we plot the expectation value of $u_{k}^{2}+v_{k}^{2}$ as a function of lattice size. The general tendencies identified for the action can be seen also here. The results of contour 0 are slightly better now, presumably since no higher order terms are involved.

In figure 4 we plot the mean phase factor as a function of lattice size. One can see that on this logarithmic scale it decreases linearly for all contours (the lines plotted are 


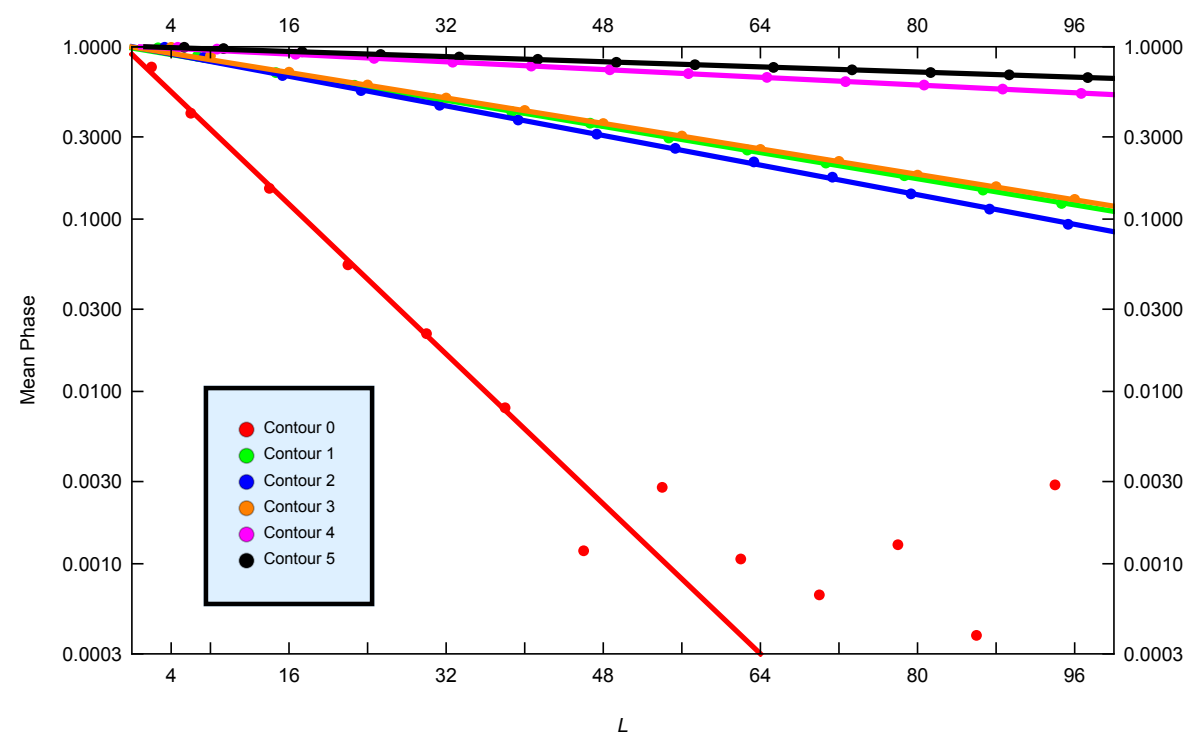

Figure 4. The mean phase factor as a function of lattice size on a logarithmic scale for the different contours together with their linear least square fits. The various contours are colour marked. Different contours are separated horizontally for clarity.

mean square fits of the data), until, for contour 0 , it gets to the range, in which we already found out that the sign problem kicks in. This loss of linear behaviour (on a logarithmic scale) that signifies the sign problem happens around a mean phase of about 0.01. It is natural to anticipate that, at least for a given theory, similar values of the mean phase factor would be associated with the sign problem for different integration contours. Thus, by extrapolating the plotted lines to 0.01 one can attempt to predict the onset of the sign problem for the other contours. We therefore expect that the onset of the sign problem would occur around $L=200$ for contour $1,2,3$, around $L=700$ for contour 4 , and around $L=1100$ for contour 5 .

In order to examine the suggested rule of thumb, we simulated contour 1,2,3 for $L=150,200, \ldots, 500$. The results are shown in figure 5 together with the previously found fit. The exponential decrease of the mean phase factor continues until about $L=200$, where it begins to fluctuate. In some cases the mean phase becomes negative and so it cannot be presented on the logarithmic scale used. Around the same value of $L$ the action density begins to fluctuate and to develop large standard errors. Yet another (unplotted) consequence of the emergence of the sign problem is the appearance of large values of the imaginary part of the observables. All that illustrates our prediction for this case. Next, we examine the same predictions for contour 4 (we do not examine contour 5 , for which we do not have a fast algorithm). The results are presented in figure 6 . The behaviour is essentially the same and we conclude that the rule of thumb suggested holds in this case as well. Such estimates can be very useful, since they can enable one to understand which $(p, q)$ order to pick for obtaining reliable results on a given large lattice, by simulating other contours on much smaller lattices. 

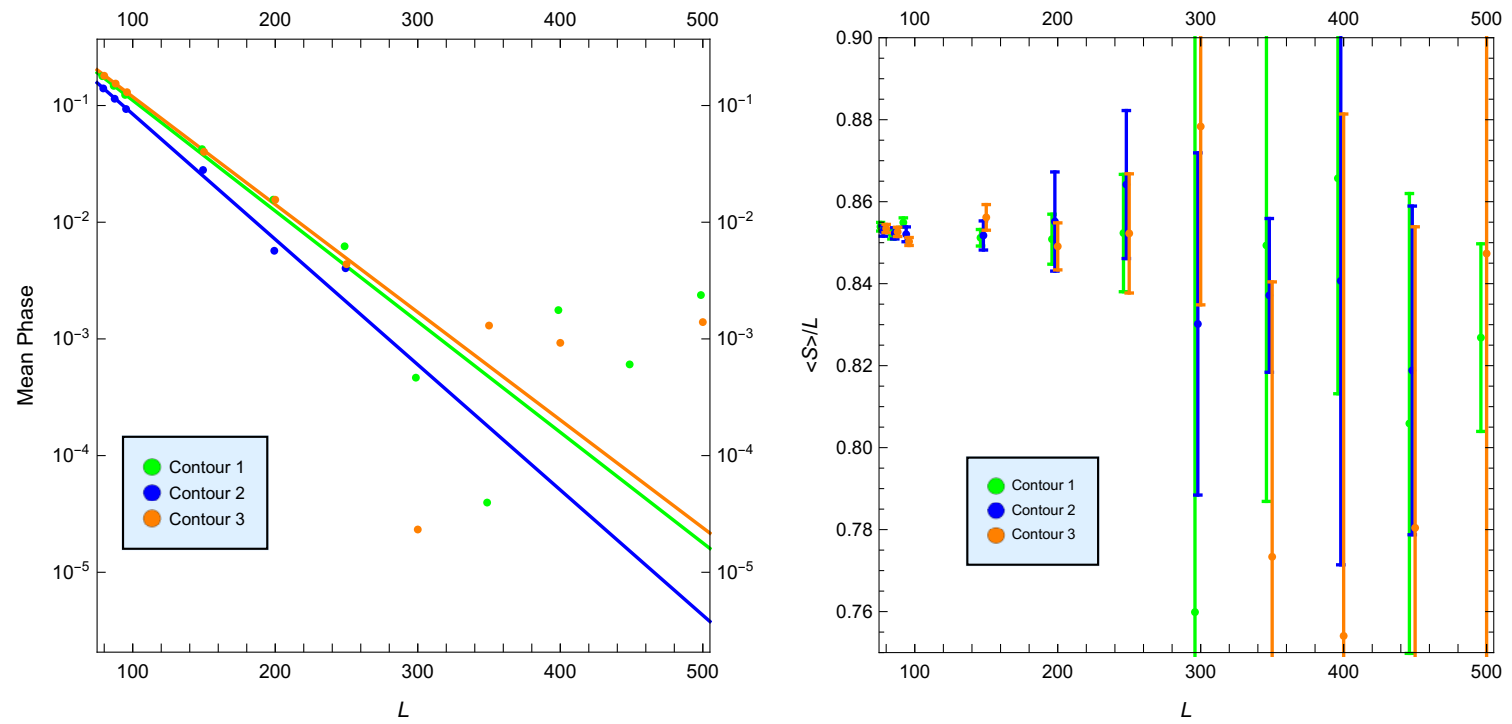

Figure 5. The onset of the sign problem for contours 1,2,3. Left: the mean phase as a function of lattice size on a logarithmic scale together with the previously obtained linear fits. Right: the action density as a function of lattice size. The various contours are colour marked. Different contours are separated horizontally for clarity.
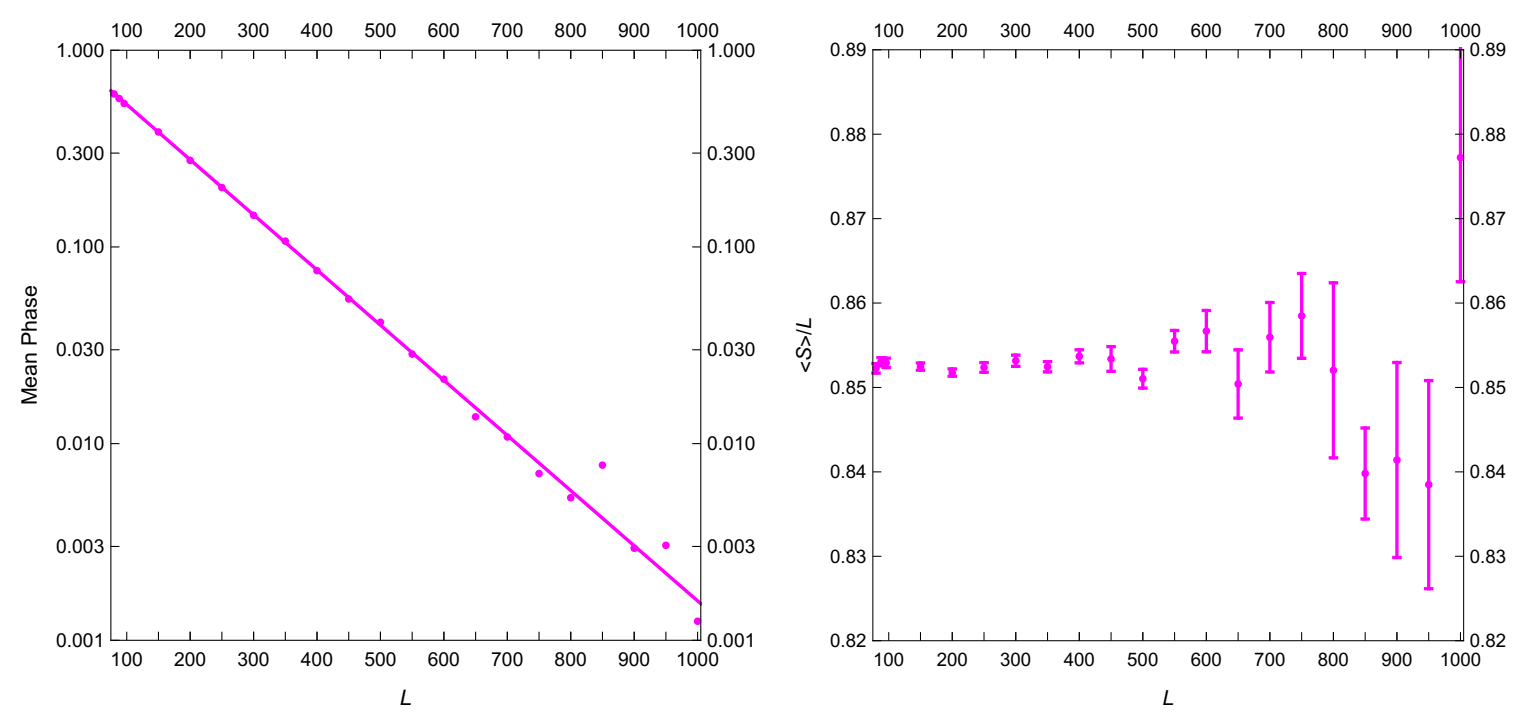

Figure 6. The onset of the sign problem for contour 4. Left: the mean phase as a function of lattice size on a logarithmic scale together with the previously obtained linear fit. Right: the action density as a function of lattice size. 


\subsection{Resolving the numerical instability}

As mentioned above, contour 4 suffers from numerical instability when simulated using the fast algorithm that relies on (4.13). Four possible resolutions to the stability problem where offered at the end of section 4. To these options we add "option 0", of completely ignoring the problem. We rewrite our options below and refer again to the end of section 4 for details:

0. Do nothing.

1. Use LU decomposition; this is exact and numerically robust but slow.

2. Change the parameters to avoid the problem.

3. Leave the contour of one specific point undeformed.

4. Use the approximate form for the Jacobian (4.15).

We examined the same observables as above for all these options. For option 2, we looked for other local maxima (in the space of parameters) of the mean phase factor on a small lattice. In light of the form obtained for the parameters defining the original contour 4 we considered only the 4-parameter family of U(1)-invariant forward nearest neighbour contours. One of the local maxima we found did not suffer from numerical instability. The parameters of this contour are

$$
\begin{aligned}
a_{000001} & =0.82, & a_{000100} & =1.27, \\
b_{000002} & =b_{002000}=0.10, & b_{000200} & =b_{200000}=0.40 .
\end{aligned}
$$

We use these parameters for defining option 2 in what follows. For option 3, we verified that no anomalous behaviour is obtained at the special point.

In figure 7 the results for the action obtained by the different options are compared. We see that the "approximate" option 4, based on (4.15), is in fact exact beyond a given small critical value $L_{c}$. Hence, in the previous subsection, we used the exact slow algorithm up to $L_{c}$ and the "approximate" one from this value on. One can still wonder why is the approximation so good and whether this would necessarily remain the case for all values of $L>L_{c}$. It turns out that, within our working precision, this approximation is exact throughout this range. The reason for that can be traced to the values that can be obtained by the matrices $S_{k}$ (4.9), from which the matrix $M$ is constructed. It is hard to evaluate these values analytically, but we found out that throughout the run, the norm of each row or column vector, within the $S_{k}$, never gets values above 0.631. Then, using Cauchy-Schwarz inequality, the absolute value of all entries of $S_{k} S_{k+1}$ cannot exceed $0.631^{2}=0.398$. Then, the entries of $S_{k} S_{k+1} S_{k+2} S_{k+3}$ cannot exceed $2 \times 0.398^{2}=0.317$. Continuing this way, one can deduce that as $L$ grows the possible maximal absolute value of the entries of $M$ becomes smaller and smaller, until for some critical value $L_{c}$ it gets below our precision threshold. Further increasing $L$ can only improve the approximation. Note that the numbers written above can only be given for fixing an upper bound on $L_{c}$. From the simulations we actually find that it is lower than this upper bound. 


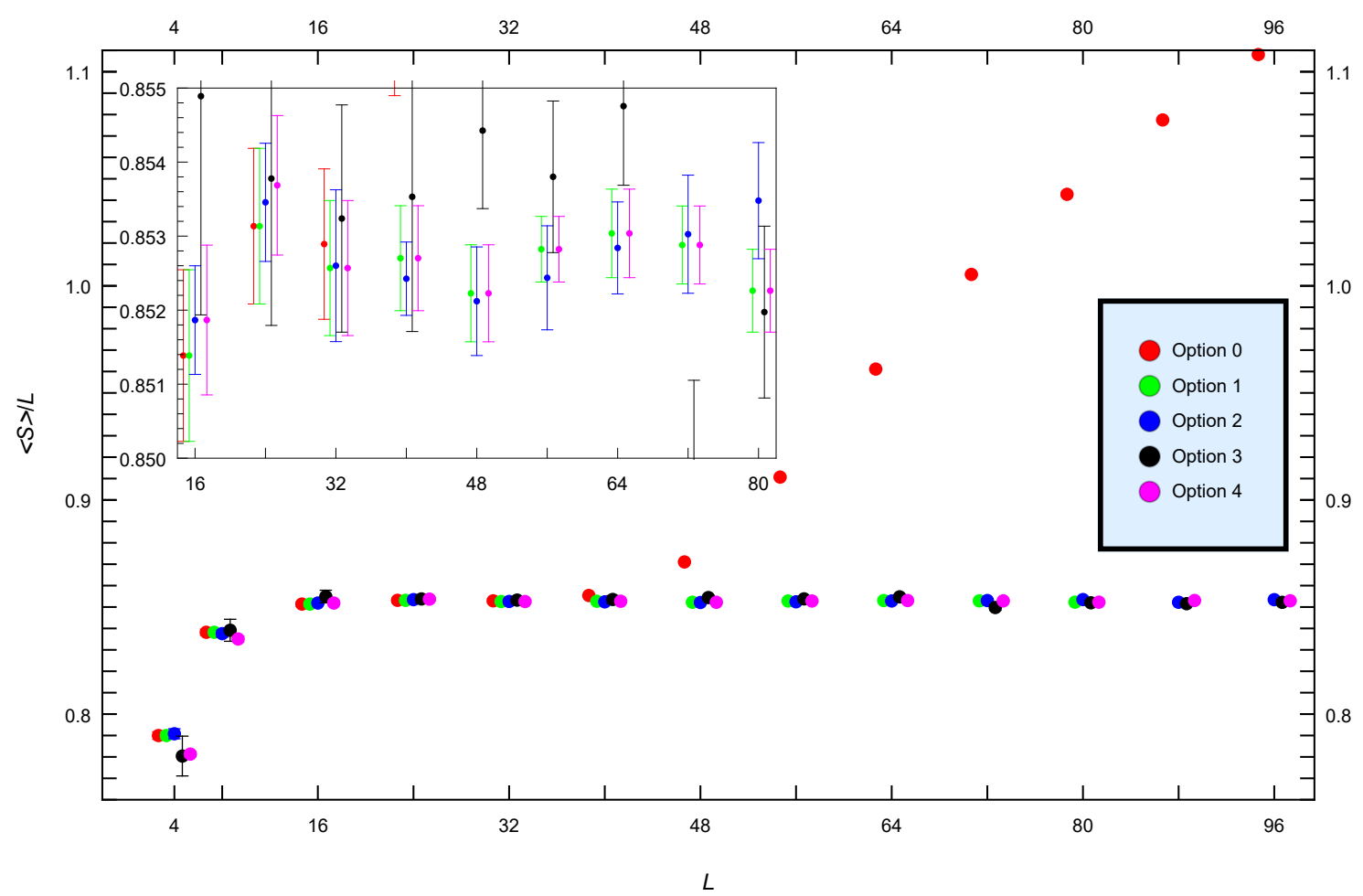

Figure 7. The action density for the different options that can be used for resolving the numerical stability of contour 4 . The various options are colour marked. Ignoring the problem (red) leads to an erroneous increase of the action density beyond $L \simeq 40$. All other options lead to similar and consistent results. As can be seen in the inset, in which the $y$ coordinate is magnified, for $L>L_{c} \equiv 24$ option 4 (using the approximate expression for the Jacobian) produces similar results to the ones obtained by option 1 (using the exact algorithm). In fact, the two options produce in this range identical results, within our working precision.

While using option 4 resolves the problem in the case at hand, it is still worth comparing the mean phase in all the cases, since in the general case the actual numbers might be different and it is important to know how the other options could be of help. In figure 8 the mean phase as a function of lattice size is presented for the various options. We see that the phase of option 0 does not decay exponentially in the range in which this is expected to happen, i.e. the fit in this case is not particularly convincing. This is another indication that something is wrong with this approach. We use again the rule of thumb for predicting when the sign problem would kick in for the various other cases. Extrapolating the numerical fits drawn in figure 8 suggest that while options 1 and 4 (the "contour 4" of the previous subsection) are expected to give reliable results up to about $L=700$, option 2 should be reliable up to about $L=400$, while option 3, which appears to be much lower than all the other ones in the plot, would give consistent results up to about $L=500$. The reason for this relative success of this approach is that, as expected, the slope in this case is almost identical to the slope of the usual contour 4 ( -0.00645 for contour 4 as compared to -0.00650 for option 3 on the logarithmic scale). The difference between the two cases is related to the large constant phase obtained from the one point whose contour was not 


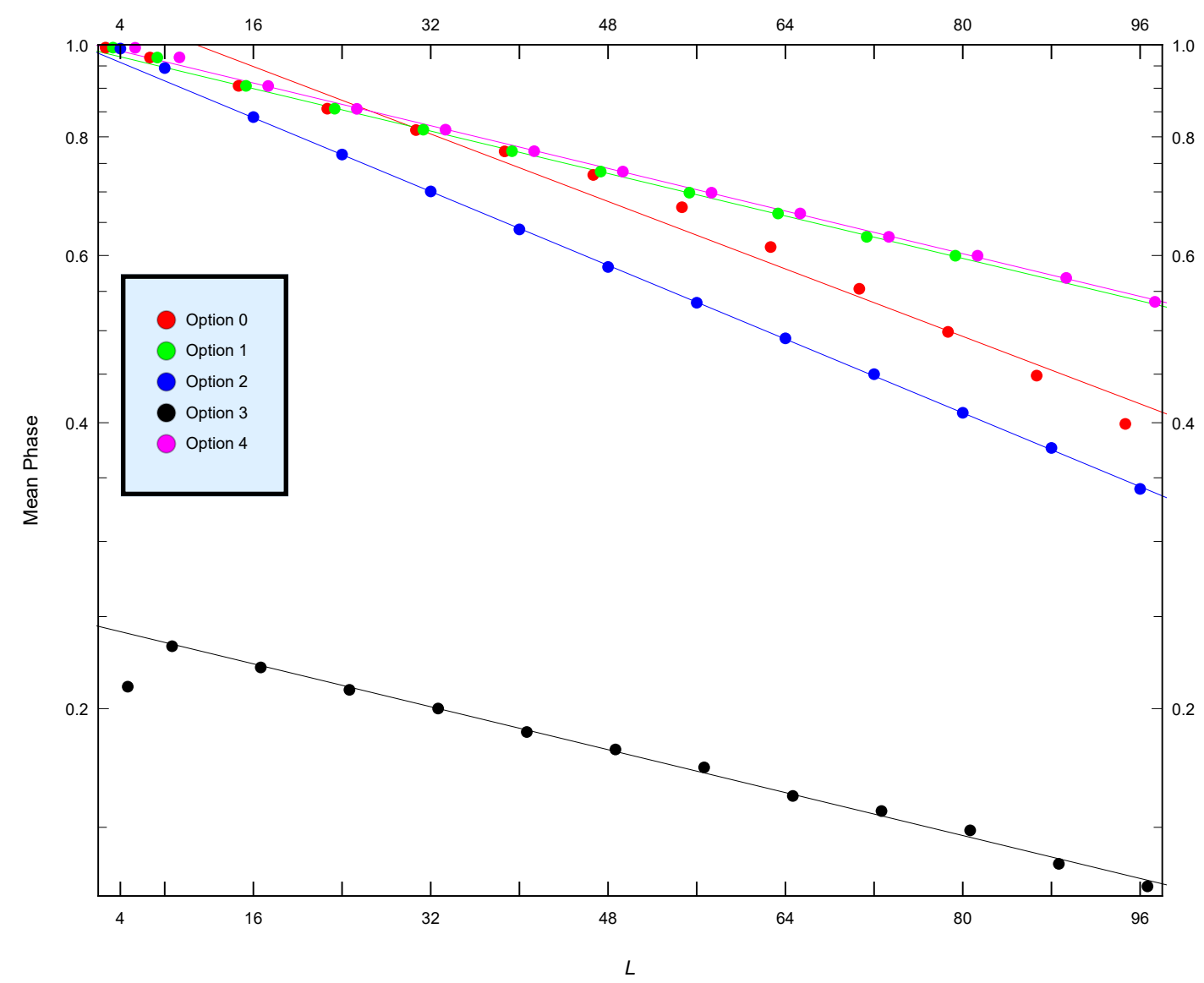

Figure 8. The mean phase as a function of lattice size on a logarithmic scale for the different options for resolving the numerical stability of contour 4 together with their linear least square fits. The various options are colour marked and are separated horizontally for clarity.

deformed. We deduce that in cases in which one cannot claim, as we do here, that the approximation (4.15) is exact, treating one point differently from the others would probably be the best approach. Moreover, one can easily increase the initial low phase in this case by allowing a deformation of the contour in the special point that would be strictly local (that is, without a dependence on any nearest neighbour).

\section{Discussion}

In this work we illustrated, using a toy model, a viable method for addressing the sign problem. The method relies on several simple principles, sketched in the introduction (section 1). We demonstrated that with a relatively low number of configurations $(300,000)$ and with a small number of free parameters, one can reliably simulate lattices of not too small size. We also suggested how to generalise our construction in a way that should give reliable lattice simulations also on quite large lattices. While the generalisation to other physical systems would require repeating the steps used in the paper in order to construct an adequate ansatz, this construction is relatively simple in principle and presumably can be 
performed for various systems. The parameters describing the suggested contours depend on the values of the lattice parameters, but their small number together with the ability to find them on a small lattice, suggest that they can be easily found at the beginning of a simulation.

Our method leads to a significant improvement in running times. While we estimated in the introduction that at $L=96$ the running time for contour 0 that suffers from a sign problem would be at least a couple of months on a standard laptop, our actual running time was much shorter and led to more accurate results: without imposing the fast evaluation of the Jacobian, described in section 4 , the running time for $L=96$ was about one week, while using the fast evaluation it was reduced to about two minutes! Results at $L=1000$ were obtained within about 20 minutes. Longer running times on fast computers would enable obtaining results for very large lattices.

While the introduction of a fast algorithm for simulating forward nearest neighbours certainly helps, one could still worry about the fact that only one nearest neighbour could be included. This is not quite so. One could extend our expressions and allow the $y_{k}$ and $\zeta_{k}$ to depend on the fields at $k, k+1, k+2, \ldots$, etc. This would lead to almost uppertriangular matrices. The evaluation of the determinant could then be performed either by setting some of the contours to the trivial ones, or by generalising the ideas presented in section 4 . In this way the approach can be extended to work also for terms induced by the secondary interaction of next to nearest neighbours, etc.

Another possible reservation would concern the generalisation to higher dimensions: it seems that when lattice points are coupled to neighbours from different space-time directions one would not be able to obtain the almost upper-block-triangular matrix needed for the fast evaluation of the Jacobian. Again, there are several ways out:

1. At any number of dimensions the 0 direction is special, since this is the direction related to the chemical potential. Including only nearest neighbour terms in this direction would already take care of the most significant contribution to the sign problem.

2. One could generalise the prescription of option 3 of subsection 5.3, such that there would be no deformations of the contour for lattice points that lie along specific co-dimension 1 hyper-surfaces (one could allow some restricted deformations of the contour at these points). This would give an upper-block-triangular matrix. There is a drawback to this approach: since the number of points in the co-dimension one surface goes like $L^{d-1}$, neglecting to deform them could lead to a significant sign problem by itself. Thus, this is probably not the best option for $d>1$.

3. One could split the lattice points such that some points would be coupled to neighbours in the 0 direction and some others to neighbours in other directions, in a way that would still produce an almost upper-block-triangular matrix. The approximate continuity of the field on the lattice (enforced by the kinetic term) would induce an effective coupling of all fields in all directions.

4. One could generalise the method introduced here such that some non-locality would be allowed, but in a way that could still be evaluated using a fast algorithm. 
5. One could rewrite the action before summation such that the points near the boundary would be treated differently. Then, when we impose the requirement that the terms vanish even before summation, different equations would be obtained for these points, in a way that is consistent with the forward nearest neighbour prescription.

6. There is always the option of using a slow algorithm for evaluating the Jacobian. For some systems there is a bottleneck of $O\left(L^{4}\right)$ even without a sign problem, coming, e.g. from a fermionic determinant. For such systems there is no reason to look for fast algorithms for the evaluation of the Jacobian. Moreover, if all else fails for a system without such a bottleneck, one could still use the slow algorithm, since while being slow compared to the $O(L)$ algorithm, it is still polynomial with not a very high power.

We currently examine variants of all these possibilities and hope to describe the results in a future work.

Another important observation regarding generalisations to higher dimensions is the following one: our approach is based on an expansion around $\alpha=0$. In light of the definition of $\alpha$ (1.4) we interpreted this as an expansion around the infinite mass limit. However, one could also think of the expansion as being around the $d \rightarrow \infty$ limit. Hence, it would be natural to expect that for a fixed number of lattice points and fixed values of the parameters, our approach would work better for large $d$ than for small $d$. In particular, we expect that for the current model our approach would deal better with the sign problem for $d=2,3,4$ than for the current $d=1$ case. Given the fact that we could get up to almost 1,000 lattice points before the sign problem kicks in at $d=1$, we believe that we would be able to get to about $10^{4}=10,000$ lattice points at $d=4$, as in [23], where the complex Langevin method was employed. Moreover, in the current paper we considered only the first order in the $\alpha$ expansion and generalisations of its form. Going to the second order is straightforward. It could further improve the success of the approach. We currently examine these issues as well.

As mentioned, generalisation of the approach to local theories with more distant neighbours is simple. However, in the case of a non local theory, e.g. a bosonic theory obtained after the integration of fermions, implementation of the approach would be less straightforward. Since the implementation of the approach is anyway model dependent, we refrain here from offering particular directions for this case and leave this, very important, question for future work.

\section{Acknowledgments}

We would like to thank Kouji Kashiwa, Scott Lawrence, Yuto Mori, Akira Ohnishi, and Benjamin Svetitsky for discussions. The research of M.K. was supported by the Israel Science Foundation (ISF), grant No. 244/17 and by EU FP7 IRSES program STREVCOMS, grant no. PIRSES-2013-612669.

Open Access. This article is distributed under the terms of the Creative Commons Attribution License (CC-BY 4.0), which permits any use, distribution and reproduction in any medium, provided the original author(s) and source are credited. 


\section{References}

[1] P. de Forcrand, Simulating QCD at finite density, PoS(LAT2009)010 (2009) [arXiv: 1005.0539] [INSPIRE].

[2] G. Aarts, Introductory lectures on lattice QCD at nonzero baryon number, J. Phys. Conf. Ser. 706 (2016) 022004 [arXiv: 1512.05145] [INSPIRE].

[3] L. Medina and M.C. Ogilvie, Simulation of scalar field theories with complex actions, arXiv:1712.02842 [INSPIRE].

[4] C.M. Bender and S. Boettcher, Real spectra in non-Hermitian Hamiltonians having PT symmetry, Phys. Rev. Lett. 80 (1998) 5243 [physics/9712001] [INSPIRE].

[5] C.M. Bender, Making sense of non-Hermitian Hamiltonians, Rept. Prog. Phys. 70 (2007) 947 [hep-th/0703096] [INSPIRE].

[6] F. Bursa and M. Kroyter, Lattice string field theory: The linear dilaton in one dimension, JHEP 10 (2014) 74 [arXiv: 1405.5089] [INSPIRE].

[7] E. Witten, Noncommutative geometry and string field theory, Nucl. Phys. B 268 (1986) 253 [INSPIRE].

[8] N. Metropolis, A.W. Rosenbluth, M.N. Rosenbluth, A.H. Teller and E. Teller, Equation of state calculations by fast computing machines, J. Chem. Phys. 21 (1953) 1087 [INSPIRE].

[9] R.V. Gavai and S. Gupta, Pressure and nonlinear susceptibilities in QCD at finite chemical potentials, Phys. Rev. D 68 (2003) 034506 [hep-lat/0303013] [INSPIRE].

[10] P. de Forcrand and O. Philipsen, The QCD phase diagram for small densities from imaginary chemical potential, Nucl. Phys. B 642 (2002) 290 [hep-lat/0205016] [INSPIRE].

[11] M. D'Elia and M.-P. Lombardo, Finite density QCD via imaginary chemical potential, Phys. Rev. D 67 (2003) 014505 [hep-lat/0209146] [INSPIRE].

[12] G. Aarts, Complex Langevin dynamics and other approaches at finite chemical potential, PoS (LATTICE2012) 017 (2012) [arXiv: 1302.3028] [INSPIRE].

[13] G. Aarts, E. Seiler and I.-O. Stamatescu, The Complex Langevin method: When can it be trusted?, Phys. Rev. D 81 (2010) 054508 [arXiv: 0912.3360] [InSPIRE].

[14] G. Aarts, F.A. James, E. Seiler and I.-O. Stamatescu, Complex Langevin: etiology and diagnostics of its main problem, Eur. Phys. J. C 71 (2011) 1756 [arXiv:1101.3270] [INSPIRE].

[15] K. Nagata, J. Nishimura and S. Shimasaki, Argument for justification of the complex Langevin method and the condition for correct convergence, Phys. Rev. D 94 (2016) 114515 [arXiv: 1606.07627] [INSPIRE].

[16] G. Guralnik and Z. Guralnik, Complexified path integrals and the phases of quantum field theory, Annals Phys. 325 (2010) 2486 [arXiv:0710.1256] [INSPIRE].

[17] E. Witten, Analytic continuation of Chern-Simons theory, AMS/IP Stud. Adv. Math. 50 (2011) 347 [arXiv:1001.2933] [INSPIRE].

[18] AuroraScience collaboration, M. Cristoforetti et al., New approach to the sign problem in quantum field theories: High density QCD on a Lefschetz thimble, Phys. Rev. D 86 (2012) 074506 [arXiv: 1205.3996] [INSPIRE]. 
[19] A. Alexandru, G. Başar, P.F. Bedaque, G.W. Ridgway and N.C. Warrington, Fast estimator of Jacobians in the Monte Carlo integration on Lefschetz thimbles, Phys. Rev. D 93 (2016) 094514 [arXiv: 1604.00956] [INSPIRE].

[20] A. Alexandru, G. Başar and P. Bedaque, Monte Carlo algorithm for simulating fermions on Lefschetz thimbles, Phys. Rev. D 93 (2016) 014504 [arXiv: 1510.03258] [INSPIRE].

[21] A. Alexandru, G. Başar, P.F. Bedaque, G.W. Ridgway and N.C. Warrington, Monte Carlo calculations of the finite density Thirring model, Phys. Rev. D 95 (2017) 014502 [arXiv: 1609.01730] [INSPIRE].

[22] P.F. Bedaque, A complex path around the sign problem, EPJ Web Conf. 175 (2018) 01020 [arXiv: 1711.05868] [INSPIRE].

[23] G. Aarts, Can stochastic quantization evade the sign problem? The relativistic Bose gas at finite chemical potential, Phys. Rev. Lett. 102 (2009) 131601 [arXiv:0810.2089] [INSPIRE].

[24] Y. Mori, K. Kashiwa and A. Ohnishi, Toward solving the sign problem with path optimization method, Phys. Rev. D 96 (2017) 111501 [arXiv:1705.05605] [INSPIRE].

[25] Y. Mori, K. Kashiwa and A. Ohnishi, Application of a neural network to the sign problem via the path optimization method, PTEP 2018 (2018) 023B04 [arXiv:1709.03208] [INSPIRE].

[26] A. Alexandru, P.F. Bedaque, H. Lamm and S. Lawrence, Finite-density Monte Carlo calculations on sign-optimized manifolds, Phys. Rev. D 97 (2018) 094510 [arXiv: 1804.00697] [INSPIRE].

[27] A. Alexandru, P.F. Bedaque, H. Lamm, S. Lawrence and N.C. Warrington, Fermions at finite density in 2+1 dimensions with sign-optimized Manifolds, Phys. Rev. Lett. 121 (2018) 191602 [arXiv: 1808.09799] [INSPIRE].

[28] S. Lawrence, Beyond thimbles: sign-optimized manifolds for finite density, in 36th International Symposium on Lattice Field Theory (Lattice 2018), East Lansing, MI, U.S.A., July 22-28, 2018 (2018) [arXiv: 1810.06529] [INSPIRE]. 